\title{
Topcolor breaking through boundary conditions
}

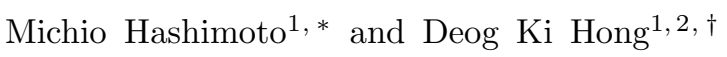 \\ ${ }^{1}$ Department of Physics, Pusan National University, Pusan 609-735, Korea \\ ${ }^{2}$ Center for Theoretical Physics, Massachusetts Institute of Technology, Cambridge, MA 02139, USA
}

(Dated: November 15, 2018)

\begin{abstract}
The nontrivial boundary conditions (BC's) for the Topcolor breaking are investigated in the context of the $\mathrm{TeV}$-scale extra dimension scenario. In the gauge symmetry breaking mechanism via the BC's we do not need to incorporate a dynamical mechanism for the Topcolor breaking into the model. Moreover, the Topcolor breaking can be realized without introducing explicitly a (composite) scalar field. We present a six dimensional model where the top and bottom quarks in the bulk have the Topcolor charge while the other quarks in the bulk do not. We also put the electroweak gauge interaction in the six dimensional bulk. The bottom quark condensation is naturally suppressed owing to the power-like running of the bulk $U(1)_{Y}$ interaction, so that only the top condensation is expected to take place. We explore such a possibility based on the ladder Schwinger-Dyson equation and show the cutoff to make the model viable.
\end{abstract}

PACS numbers: 11.15.Ex,11.10.Kk,11.25.Mj,12.60.Rc

\section{INTRODUCTION}

Recently, the model building along with the TeV-scale extra dimension scenario [1, 2] has been widely surveyed. The gauge theories with extra dimensions have remarkable features. Since the number of the Kaluza-Klein $(\mathrm{KK})$ modes which is the source of the attractive force increases explosively in high-energy, the bulk gauge couplings grow very quickly and get strong. Therefore the bulk gauge theories can naturally trigger the dynamical chiral symmetry breaking (D $\chi \mathrm{SB})$. 3, 4, 5, 6, 7, 8]

The top quark condensate $[9,10,11,12$, which is often called the "top mode standard model" (TMSM), has been also reexamined in the context of extra dimensions [3, 4, 5, 6, 13, 14, 15, 18. In particular, ArkaniHamed, Cheng, Dobrescu and Hall (ACDH) 5] proposed a version of the TMSM where the third generation quarks and leptons as well as the the Standard Model (SM) gauge bosons are put in the bulk, while any four-fermion interactions are not introduced in the bulk unlike the original version of the TMSM in four dimensions. In Refs. [6, 14], the full bulk gauge dynamics was investigated, based on the ladder Schwinger-Dyson (SD) equation. The phenomenological implications were studied in Ref. [15]. It is found that the model with $D=8$ can be viable and both masses of the top quark and Higgs boson are predicted as $m_{t}=172-175 \mathrm{GeV}$ and $m_{H}=176-188$ $\mathrm{GeV}$, respectively. However it turns out that the simplest scenario with $D=6$ does not work.

On the other hand, it is known that field theories in six dimensions have several interesting features relating to proton stability [16], explanation of the number of the

\footnotetext{
*E-mail: michioh@charm.phys.pusan.ac.kr, mhashimo@uwo.ca The present address is Department of Applied Mathematics, Western Science Centre, The University of Western Ontario, London ON Canada N6A 5B7

${ }^{\dagger}$ E-mail: dkhong@pusan.ac.kr, dkhong@lns.mit.edu
}

generations of fermions [17], etc.. In order to construct a viable top-condensate model in six dimensions, we may introduce the four-fermion interaction in the bulk. In other words, one of possibilities is the model building based on the gauged Nambu-Jona-Lasinio (NJL) model which is defined as the gauge theory with four-fermion interactions. The phase structure of such a gauged NJL model in the bulk was analyzed in Ref. [18]. Another possibility is to introduce a strong gauge interaction such as Topcolor in the bulk. Topcolor models in four dimensions have been extensively studied. 19, 20, 21] (See for reviews Refs. 22, 23]. ) The top seesaw mechanism can be realized by introducing five dimensional right-handed top quark. [4, 24, 25]

The Topcolor should be broken down in low energy. In four dimensions, however, it is required to introduce some involved dynamical mechanism in order to break the Topcolor, unless a (composite) scalar field is introduced for simplicity. As for the gauge symmetry breaking, the extra dimension scenario has an advantage as well. It is known that the gauge symmetry breaking can be easily achieved in extra dimensions by imposing appropriate boundary conditions (BC's). [26] On the basis of more general BC's, the Higgsless theory was proposed [27, 28] and has been investigated by a number of authors 29, 30, 31, 32, 33, 34, 35, 36, 37, 38, 39, 40, 41. Although it may be difficult to construct Higgsless models consistent with the precision measurements, the gauge symmetry breaking mechanism via nontrivial BC's can be also applied to other models for the dynamical electroweak symmetry breaking. Such an attempt has not yet been discussed so far.

A Topcolor model with nontrivial BC's obviously has some advantages: We do not need to incorporate a dynamical mechanism for the Topcolor breaking. We can break spontaneously the Topcolor gauge symmetry without introducing explicitly a (composite) scalar field. We note here, however, that such a model has implicitly a scalar field on the boundary. In a sense, a theory with 
nontrivial BC's is equivalent to a model having a scalar field with a large vacuum expectation value (VEV) which is put on the boundary. In the infinite limit of the VEV the scalar field is completely decoupled and hence the KK masses of the gauge boson are controlled only by the compactification scale. Therefore, we can neglect thoroughly such a scalar field. In passing, the Topcolor gauge bosons do not have mass terms in the bulk in the gauge breaking mechanism via the BC's. The theory thus does not provide four-fermion (NJL-type) interactions in the bulk, unlike four dimensional Topcolor models.

In this paper we investigate the Topcolor breaking via nontrivial BC's in six dimensions. We assign the Topcolor charge, $S U(3)_{1}$, to the top and bottom quarks in the bulk. The quarks of the first and second generations in the bulk are assumed to have the $S U(3)_{2}$ charge. We then impose the nontrivial BC's so that $S U(3)_{1} \times S U(3)_{2}$ breaks down to the diagonal subgroup, which is identified to QCD. We also put the electroweak (EW) gauge interaction in the bulk. The EW gauge sector is the same as the universal extra dimension model [42] with the composite Higgs field. In order to obtain the chiral fermion in four dimensions, we apply the compactification on a square proposed by Dobrescu and Pontón 43], which is closely related to the compactification on the orbifold $T^{2} / Z_{4}$.

For a viable model it is required that only the top condensation occurs while other condensations such as bottom and leptons do not. We call the requirement "tMAC condition" and the energy scale "tMAC scale" as in Ref. 15. Once we specify the model, the renormalization group (RG) flows of the gauge couplings can be determined through the truncated KK effective theory 2. The running effects are very important to study the tMAC scale. We here note that the bulk hypercharge interaction $U(1)_{Y}$ rapidly becomes strong owing to the power-like running. Thus the $U(1)$ tilting mechanism to suppress the bottom quark condensation is automatically incorporated in the model. The difference of the gauge coupling strengths between $S U(3)_{1}$ and $S U(3)_{2}$ leads to suppression of the up-, and charmcondensations. When the theory behaves as a walking gauge theory [44, 45, 46, 47, 48] and the gauge coupling of $S U(3)_{1}$ is very close to the critical coupling for the $\mathrm{D} \chi \mathrm{SB}$, the situation that only the top quark condenses is naturally realized. We analyze the tMAC scale by using the ladder SD equation and depict the results in two dimensional plane of the cutoff $\Lambda$ and the ratio of the Topcolor and QCD couplings $g^{2}\left(R^{-1}\right) / g_{3}^{2}\left(R^{-1}\right)$ at the compactification scale $R^{-1}(\approx 10 \mathrm{TeV})$. For a slice $g^{2}\left(R^{-1}\right) / g_{3}^{2}\left(R^{-1}\right)=4.6$, for example, we find that the tMAC scale is $\Lambda R \sim 10-10.5$. We also show that the model is not excluded by constraints of $\mathrm{S}, \mathrm{T}$-parameters.

The paper is organized as follows: In Sec. [I] we study the BC's for the Topcolor breaking. In Sec. III we give the model and study running effects of gauge couplings. In Sec. IV we determine the tMAC scale by solving the ladder SD equation. Sec. $\nabla$ is devoted to summary and discussions. In Appendix $\mathrm{A}$ we give the chiral compactification on the square. In Appendix B we describe the condition that the five dimensional gauge symmetry is restored on the boundaries.

\section{BOUNDARY CONDITIONS FOR TOPCOLOR BREAKING}

We explore possibility of the top quark condensate in six dimensions. For simplicity, we compactify extra two spatial dimensions $\left(y^{5}, y^{6}\right)$ on a square with $0 \leq y^{5}, y^{6} \leq$ $L$. We introduce the bulk Topcolor gauge interaction in order to trigger the top condensation. The Topcolor should be broken down in the low-energy effective theory. In this section, we study nontrivial BC's for the Topcolor breaking. First, we derive the BC's for the pure gauge theory in the bulk. Next, we incorporate the top quark in the bulk.

\section{A. Pure gauge theory on a square}

Let us analyze the $S U(3)_{1} \times S U(3)_{2}$ gauge theory in the bulk. We assign the Topcolor to the $S U(3)_{1}$ gauge interaction. The action is given by

$$
S=\int d^{4} x \int_{0}^{L} d y^{5} \int_{0}^{L} d y^{6} \mathcal{L}_{g}
$$

with the Lagrangian

$$
\mathcal{L}_{g}=-\frac{1}{4} F_{M N}^{a} F^{a M N}-\frac{1}{4} F_{M N}^{\prime a} F^{\prime a M N},
$$

where $M=0,1,2,3,5,6$, and

$$
F_{M N}^{a} \equiv \partial_{M} A_{N}^{a}-\partial_{N} A_{M}^{a}+g_{6 D} f^{a b c} A_{M}^{b} A_{N}^{c} .
$$

$f^{a b c}$ is the structure constant of the gauge group, $g_{6 D}$ the dimensionful bulk gauge coupling constant. The definition of $F_{M N}^{\prime a}$ is the same as Eq. (II.3) with $A_{M}^{\prime a}$ and $g_{6 D}^{\prime}$. The gauge fields $A_{M}^{a}$ and $A_{M}^{\prime a}$ are associated with the gauge groups $S U(3)_{1}$ and $S U(3)_{2}$, respectively. We also use the notation

$$
A_{M} \equiv A_{M}^{a} T^{a},
$$

with $T^{a}$ being the generator of the SU(3) Lie algebra.

We break the gauge symmetry $S U(3)_{1} \times S U(3)_{2}$ to the diagonal subgroup by assigning nontrivial BC's to the gauge fields. The unbroken subgroup is identified to the conventional QCD.

After integration by parts the variation of the action 
(II.1) yields

$$
\begin{aligned}
& \delta S= \\
& \left.\begin{array}{c}
\delta d^{4} x \int d y^{5} d y^{6}\left\{\left[\partial_{M} F^{a M N}-g_{6 D} f^{a b c} F^{b M N} A_{M}^{c}\right] \delta A_{N}^{a}\right. \\
+\left[\partial_{M} F^{\prime} a M N-g_{6 D}^{\prime} f^{a b c} F^{\prime b M N} A_{M}^{\prime}\right]
\end{array} \delta A_{N}^{\prime a}\right\} \\
& +\left.\int d^{4} x \int d y^{6}\left(F_{5 \mu}^{a} \delta A^{a \mu}+F_{5 \mu}^{\prime a} \delta A^{\prime a \mu}\right)\right|_{\left(0, y^{6}\right)} ^{\left(L, y^{6}\right)} \\
& +\left.\int d^{4} x \int d y^{5}\left(F_{6 \mu}^{a} \delta A^{a \mu}+F_{6 \mu}^{\prime a} \delta A^{\prime a \mu}\right)\right|_{\left(y^{5}, 0\right)} ^{\left(y^{5}, L\right)} \\
& =0,
\end{aligned}
$$

where

$$
\left.X\right|_{(0, y)} ^{(L, y)} \equiv X\left(x^{\mu}, L, y\right)-X\left(x^{\mu}, 0, y\right),
$$

and similar is the definition of $\left.X\right|_{(y, 0)} ^{(y, L)}$. The vanishing requirement of the first term in Eq. (II.5) corresponds to the equation of motion.

The zero modes of the gauge scalar fields $A_{5,6}^{\left({ }^{\prime}\right)}$ should be eliminated from the low-energy spectrum. We thus impose the Dirichlet BC's on the gauge scalars,

$$
\left\{\begin{array}{l}
A_{5}(0, y)=A_{5}(L, y)=0, \quad A_{5}^{\prime}(0, y)=A_{5}^{\prime}(L, y)=0, \\
A_{6}(y, 0)=A_{6}(y, L)=0, \quad A_{6}^{\prime}(y, 0)=A_{6}^{\prime}(y, L)=0,
\end{array}\right.
$$

where we abbreviated the trivial argument $x^{\mu}$ in $A_{5}\left(x^{\mu}, 0, y\right)$, etc. We rewrite the two integrals $\int d y^{6}, \int d y^{5}$ in Eq. gle dummy index $y$. Then we obtain a $\mathrm{BC}$ for $A_{\mu}^{a}$ and $A_{\mu}^{\prime a}$,

$$
\begin{gathered}
\left.\left(\partial_{5} A_{\mu}^{a} \delta A^{a \mu}+\partial_{5} A_{\mu}^{\prime a} \delta A^{\prime a \mu}\right)\right|_{(0, y)} ^{(L, y)} \\
+\left.\left(\partial_{6} A_{\mu}^{a} \delta A^{a \mu}+\partial_{6} A_{\mu}^{\prime a} \delta A^{\prime a \mu}\right)\right|_{(y, 0)} ^{(y, L)}=0 .
\end{gathered}
$$

If the variations $\delta A_{\mu}^{\left({ }^{\prime}\right)}$ on the boundaries, $(0$ or $L, y)$ and $(y, 0$ or $L)$, are independent, Eq. (II.8) yields two BC's,

$$
\left.\left(\partial_{5} A_{\mu}^{a} \delta A^{a \mu}+\partial_{5} A_{\mu}^{\prime a} \delta A^{\prime a \mu}\right)\right|_{(0, y)} ^{(L, y)}=0,
$$

and

$$
\left.\left(\partial_{6} A_{\mu}^{a} \delta A^{a \mu}+\partial_{6} A_{\mu}^{\prime a} \delta A^{\prime a \mu}\right)\right|_{(y, 0)} ^{(y, L)}=0 .
$$

Since we adopt later on a chiral compactification on the square with two adjacent sides identified, we use a general expression (II.8) in the following discussion.
Now we further assign the following $\mathrm{BC}$ to $A_{\mu}$ and $A_{\mu}^{\prime}$ on every boundary,

$$
\left.A_{\mu}\right|^{(0, y),(L, y),(y, 0),(y, L)}=\left.\tan \theta A_{\mu}^{\prime}\right|^{(0, y),(L, y),(y, 0),(y, L)},
$$

where $\theta$ is a constant. Note that the derivative terms are not identified at the boundary, i.e., $\left.\partial_{5} A_{\mu}\right|^{(0, y)} \neq$ $\left.\tan \theta \partial_{5} A_{\mu}^{\prime}\right|^{(0, y)}$, etc. The BC (11.8) is then rewritten as

$$
\begin{gathered}
\left.\partial_{5}\left[A_{\mu}^{\prime a}+\tan \theta A_{\mu}^{a}\right] \delta A^{\prime a \mu}\right|_{(0, y)} ^{(L, y)} \\
+\left.\partial_{6}\left[A_{\mu}^{\prime a}+\tan \theta A_{\mu}^{a}\right] \delta A^{\prime a} \mu\right|_{(y, 0)} ^{(y, L)}=0 .
\end{gathered}
$$

We here define the "gluon" field $G_{\mu}$ and the "coloron" field $G_{\mu}^{\prime}$ as

$$
\left\{\begin{array}{l}
G_{\mu}\left(x^{\mu}, y^{5}, y^{6}\right)=A_{\mu}^{\prime} \cos \theta+A_{\mu} \sin \theta \\
G_{\mu}^{\prime}\left(x^{\mu}, y^{5}, y^{6}\right)=-A_{\mu}^{\prime} \sin \theta+A_{\mu} \cos \theta
\end{array}\right.
$$

The gluon field should have zero modes. We thus impose the Neumann BC's on the gluon field $G_{\mu}$ :

$$
\left.\partial_{5} G_{\mu}^{a}\right|^{(0, y),(L, y)}=0,\left.\quad \partial_{6} G_{\mu}^{a}\right|^{(y, 0),(y, L)}=0 .
$$

By definition (II.13), Eq. (II.11) automatically yields the Dirichlet BC's for $G_{\mu}^{\prime}$ :

$$
G_{\mu}^{\prime}(0, y)=G_{\mu}^{\prime}(L, y)=G_{\mu}^{\prime}(y, 0)=G_{\mu}^{\prime}(y, L)=0 .
$$

Hence we obtain the KK decompositions for $G_{\mu}$ and $G_{\mu}^{\prime}$,

$$
\begin{aligned}
G_{\mu}\left(x^{\mu}, y^{5}, y^{6}\right) & =\frac{1}{L} \sum_{n_{5}, n_{6} \geq 0} G_{\mu}^{\left[n_{5}, n_{6}\right]}\left(x^{\mu}\right) \\
\times \mathcal{N} & {\left[\cos \left(\frac{\pi}{L} n_{5} y^{5}\right) \cos \left(\frac{\pi}{L} n_{6} y^{6}\right)\right], }
\end{aligned}
$$

with

$$
\mathcal{N} \equiv 2 \sqrt{\frac{1}{\left(1+\delta_{n_{5}, 0}\right)\left(1+\delta_{n_{6}, 0}\right)}},
$$

and

$$
\begin{array}{r}
G_{\mu}^{\prime}\left(x^{\mu}, y^{5}, y^{6}\right)=\frac{1}{L} \sum_{n_{5}, n_{6}>0} G_{\mu}^{\prime}{ }^{\left[n_{5}, n_{6}\right]}\left(x^{\mu}\right) \\
\times 2\left[\sin \left(\frac{\pi}{L} n_{5} y^{5}\right) \sin \left(\frac{\pi}{L} n_{6} y^{6}\right)\right],
\end{array}
$$

respectively. We here note that only $G_{\mu}$ associated with the diagonal subgroup includes a zero mode, while $G_{\mu}^{\prime}$ does not. We identify the unbroken gauge group to QCD, $S U(3)_{c}$.

We comment on our choice of the BC's. Under the above identification the gauge symmetry breaking $S U(3)_{1} \times S U(3)_{2} \rightarrow S U(3)_{c}$ takes place on all the boundaries. This is not a unique choice: for example, we can also construct a model in which the gauge symmetry is broken down only at a part of boundary like $(0, y),(L, y)$. We choose the BC's (II.14)- (II.15) to be consistent with the chiral compactification. 


\section{B. Topcolor model on a square}

Let us take into account the top quark $T$ in the bulk, which has the $S U(3)_{1}$ charge,

$$
\mathcal{L}_{t}=\bar{T}_{+} i D_{M} \Gamma^{M} T_{+}+\bar{T}_{-} i D_{M} \Gamma^{M} T_{-},
$$

with

$$
D_{M} \equiv\left(\frac{1}{2} \stackrel{\leftrightarrow}{\partial}_{M}-i g_{6 D} A_{M}\right)
$$

where

$$
\bar{T} \stackrel{\leftrightarrow}{\partial}_{M} \Gamma^{M} T \equiv \bar{T} \Gamma^{M}\left(\partial_{M} T\right)-\left(\partial_{M} \bar{T}\right) \Gamma^{M} T
$$

and $\Gamma^{M}$ 's are the Gamma matrices in six dimensions. The chiral fermions $T_{ \pm}$in the bulk are defined by

$$
T_{ \pm} \equiv \frac{1}{2}\left(1 \pm \Gamma_{\chi, 7}\right) T
$$

where $\Gamma_{\chi, 7}$ is the chirality matrix in six dimensions. The theory obviously has the chiral symmetry. We here note that the chiral fermions $T_{ \pm}$contain both of the right and left handed components, i.e.,

$$
T_{ \pm}=T_{ \pm R}+T_{ \pm L}, \quad T_{ \pm R, L} \equiv \frac{1}{2}\left(1 \pm \Gamma_{\chi, 5}\right) T_{ \pm},
$$

with the four dimensional chirality matrix $\Gamma_{\chi, 5}$. Therefore we must carry out the chiral compactification in order to obtain the SM-like top quark in low-energy.

Following Dobrescu and Pontón [4], we identify two adjacent sides as follows:

$$
(y, 0) \equiv(0, y), \quad(y, L) \equiv(L, y), \quad{ }^{\forall} \in[0, L],
$$

which is closely related to the orbifold compactification on $T^{2} / Z_{4}$. We take a notation that $T_{+R,-L}$ include the SM-like top quarks $t_{R, L}$ as the zero modes. In general, the value of a field at two identified points differs by a nontrivial phase, if a loop around the point is noncontractible. As in [3], we assign the following BC's

$$
T_{+R}(y, 0)=T_{+R}(0, y), \quad T_{+R}(y, L)=T_{+R}(L, y),
$$

to the fermion $T_{+R}$. The BC's for $T_{-L}$ are the same. For $T_{+L}$ and $T_{-R}$ different BC's should be imposed. For details, see Appendix $\mathrm{A}$ and Ref. [43]. On the other hand, for gauge fields $G_{\mu}$ and $G_{\mu}^{\prime}$ the chiral compactification further requires

$$
G_{\mu}(0, y)=G_{\mu}(y, 0), \quad G_{\mu}(y, L)=G_{\mu}(L, y),
$$

and

$$
\left.\partial_{6} G_{\mu}^{\prime}\right|^{(y, 0)}=-\left.\partial_{5} G_{\mu}^{\prime}\right|^{(0, y)},\left.\quad \partial_{6} G_{\mu}^{\prime}\right|^{(y, L)}=-\left.\partial_{5} G_{\mu}^{\prime}\right|^{(L, y)},
$$

in addition to the BC's (II.14)-(II.15). It is natural to require that on the boundaries the theory is reduced into the five dimensional one. Details are summarized in Appendix B] We then find that the desirable BC's for the derivative terms of $T$ are

$$
\left.\partial_{5} T_{+R,-L}\right|^{(0, y),(L, y)}=0,\left.\quad \partial_{6} T_{+R,-L}\right|^{(y, 0),(y, L)}=0 .
$$

We here note that Eqs. (II.7) also imply Eq. (II.28). The KK decompositions of $T_{+R,-L}, G_{\mu}$ and $G_{\mu}^{\prime}$ are obtained as

$$
T_{+R,-L}\left(x^{\mu}, y^{5}, y^{6}\right)=\frac{1}{L} \sum_{j \geq k \geq 0} T_{+R,-L}^{[j, k]}\left(x^{\mu}\right) f_{c c}^{[j, k]}\left(y^{5}, y^{6}\right),
$$

$$
\begin{aligned}
& G_{\mu}\left(x^{\mu}, y^{5}, y^{6}\right)=\frac{1}{L} \sum_{j \geq k \geq 0} G_{\mu}^{[j, k]}\left(x^{\mu}\right) f_{c c}^{[j, k]}\left(y^{5}, y^{6}\right) \\
& G_{\mu}^{\prime}\left(x^{\mu}, y^{5}, y^{6}\right)=\frac{1}{L} \sum_{j>k>0} G_{\mu}^{\prime}{ }^{[j, k]}\left(x^{\mu}\right) f_{s s}^{[j, k]}\left(y^{5}, y^{6}\right),
\end{aligned}
$$

with

$$
\begin{aligned}
f_{c c}^{[j, k]} \equiv \mathcal{N}_{c c} & {\left[\cos \left(\frac{\pi}{L} j y^{5}\right) \cos \left(\frac{\pi}{L} k y^{6}\right)\right.} \\
& \left.+\cos \left(\frac{\pi}{L} k y^{5}\right) \cos \left(\frac{\pi}{L} j y^{6}\right)\right], \\
f_{s s}^{[j, k]} \equiv \mathcal{N}_{s s}[ & \sin \left(\frac{\pi}{L} j y^{5}\right) \sin \left(\frac{\pi}{L} k y^{6}\right) \\
& \left.-\sin \left(\frac{\pi}{L} k y^{5}\right) \sin \left(\frac{\pi}{L} j y^{6}\right)\right],
\end{aligned}
$$

where $\mathcal{N}_{c c}$ and $\mathcal{N}_{s s}$ are the normalization factors given in Appendix $\mathrm{A}$ In particular, the function $f_{c c}^{[0,0]}$ for the zero mode is given by

$$
f_{c c}^{[0,0]}=1 .
$$

In our compactification, the KK masses for $G_{\mu}^{[j, k]}$ and $G_{\mu}^{\prime}{ }_{\mu}^{[j, k]}$ are labeled by integers $j$ and $k$ as

$$
M_{j, k}^{2} \equiv \frac{\pi^{2}}{L^{2}}\left(j^{2}+k^{2}\right)
$$

The lightest KK mass for $G_{\mu}$ is, as usual,

$$
M_{G} \equiv M_{1,0}=\frac{\pi}{L}
$$

However, the coloron field does not include the KK components of $(j>0, k=0)$ and $(j, k=j)$. Therefore the lowest KK mass for the coloron is given by

$$
M_{C} \equiv M_{2,1}=\frac{\sqrt{5} \pi}{L} .
$$

We also comment that the total number of KK modes for $G_{\mu}^{\prime}$ below the cutoff $\Lambda$ is slightly smaller than that 
for $G_{\mu}$. Such a difference is, however, negligible for a large $\Lambda$.

From the symmetry breaking pattern $S U(3)_{1} \times$ $S U(3)_{2} \rightarrow S U(3)_{c}$, the gauge couplings of $S U(3)_{1}$ and $S U(3)_{2}$ are not arbitrary, but they are related to the QCD coupling. Integrating the six dimensional Lagrangian over $d y^{5}$ and $d y^{6}$, we define the four dimensional theory,

$$
\mathcal{L}_{4 D} \equiv \int_{0}^{L} d y^{5} \int_{0}^{L} d y^{6} \mathcal{L}_{6 D}
$$

with

$$
\mathcal{L}_{6 D}=\mathcal{L}_{t}+\mathcal{L}_{g}
$$

By using Eqs. (11.29)-(11.31) and the definition (11.13), we find the interaction term between zero modes of the top and the gluon as

$$
\mathcal{L}_{\text {int }}=\frac{g_{6 D} \sin \theta}{L} \bar{T}_{+R,-L}^{[0,0]} \Gamma^{\mu} G_{\mu}^{[0,0]} T_{+R,-L}^{[0,0]} .
$$

We here note that the definition (1I.38) implies the relations between the six and four dimensional gauge couplings as

$$
g_{6 D}^{2}=L^{2} g^{2}, \quad g_{6 D}^{\prime 2}=L^{2} g^{\prime 2},
$$

where $g$ and $g^{\prime}$ denote the four dimensional gauge coupling constants for $S U(3)_{1}$ and $S U(3)_{2}$, respectively. Eq. (III.40) then yields the relation

$$
g_{3}=g \sin \theta
$$

where $g_{3}$ is the four dimensional QCD coupling. In the same way, we obtain a similar relation between QCD and $S U(3)_{2}$ couplings,

$$
g_{3}=g^{\prime} \cos \theta
$$

Eqs. (11.42)-(11.43) read

$$
\frac{1}{g_{3}^{2}}=\frac{1}{g^{2}}+\frac{1}{g^{\prime 2}}
$$

\section{THE MODEL}

We now incorporate all quarks and leptons of the SM into the model. We put all of gauge fields and SM fermions in the six dimensional bulk. We may introduce right-handed neutrinos in the bulk, which is not relevant in the following analysis.

Let us assign the bulk top and bottom quarks to the $S U(3)_{1}$ charge while the quarks of the first and second generations to the $S U(3)_{2}$ charge. We assume that the electroweak gauge sector is the same as the model of the universal extra dimensions 42]. We perform the chiral compactification described in Sec. III Appendix \$ and Ref. [4]. The Topcolor interaction should be sufficiently

\begin{tabular}{|c|cccc|}
\hline & $S U(3)_{1}$ & $S U(3)_{2}$ & $S U(2)_{W}$ & $U(1)_{Y}$ \\
\hline$(t, b)_{-}$ & $\mathbf{3}$ & $\mathbf{1}$ & $\mathbf{2}$ & $1 / 6$ \\
$t_{+}$ & $\mathbf{3}$ & $\mathbf{1}$ & $\mathbf{1}$ & $2 / 3$ \\
$b_{+}$ & $\mathbf{3}$ & $\mathbf{1}$ & $\mathbf{1}$ & $-1 / 3$ \\
$\left(\nu_{\tau}, \tau\right)_{-}$ & $\mathbf{1}$ & $\mathbf{1}$ & $\mathbf{2}$ & $-1 / 2$ \\
$\tau_{+}$ & $\mathbf{1}$ & $\mathbf{1}$ & $\mathbf{1}$ & -1 \\
\hline \hline$(c, s)_{-}$ & $\mathbf{1}$ & $\mathbf{3}$ & $\mathbf{2}$ & $1 / 6$ \\
$c_{+}$ & $\mathbf{1}$ & $\mathbf{3}$ & $\mathbf{1}$ & $2 / 3$ \\
$s_{+}$ & $\mathbf{1}$ & $\mathbf{3}$ & $\mathbf{1}$ & $-1 / 3$ \\
$\left(\nu_{\mu}, \mu\right)_{-}$ & $\mathbf{1}$ & $\mathbf{1}$ & $\mathbf{2}$ & $-1 / 2$ \\
$\mu_{+}$ & $\mathbf{1}$ & $\mathbf{1}$ & $\mathbf{1}$ & -1 \\
\hline \hline$(u, d)_{-}$ & $\mathbf{1}$ & $\mathbf{3}$ & $\mathbf{2}$ & $1 / 6$ \\
$u_{+}$ & $\mathbf{1}$ & $\mathbf{3}$ & $\mathbf{1}$ & $2 / 3$ \\
$d_{+}$ & $\mathbf{1}$ & $\mathbf{3}$ & $\mathbf{1}$ & $-1 / 3$ \\
$\left(\nu_{e}, e\right)_{-}$ & $\mathbf{1}$ & $\mathbf{1}$ & $\mathbf{2}$ & $-1 / 2$ \\
$e_{+}$ & $\mathbf{1}$ & $\mathbf{1}$ & $\mathbf{1}$ & -1 \\
\hline \hline$\psi_{X}$ & $\mathbf{3}$ & $\mathbf{1}$ & $\mathbf{1}$ & 0 \\
\hline
\end{tabular}

TABLE I: The charge assignment of the model.

strong to trigger the top condensation, so that we may further introduce vector-like (heavy) fermions $\psi_{X}$ having the $S U(3)_{1}$ charge in order to adjust the RG flow of $S U(3)_{1}$. We show the charge assignment in Table \

While $S U(3)_{1}$ and $S U(3)_{2}$ are vector-like, the $S U(2)_{W}$ and $U(1)_{Y}$ representations are chiral. Although the six dimensional theory is anomalous under the charge assignment in Table \ the anomalies can be cancelled out by the Green-Schwarz mechanism [49. We assume that the Green-Schwarz counterterm does not change the results in the following analysis.

Let us study running of gauge couplings in the "truncated KK" effective theory [2] based on the $\overline{\mathrm{MS}}$-scheme. In this section, we use the unit of the extra momentum $R^{-1}$ instead of $L$,

$$
R^{-1} \equiv \frac{\pi}{L}
$$

We expand bulk fields into KK modes and construct a four dimensional effective theory. Below $R^{-1}$ the renormalization group equations (RGEs) of the four dimensional gauge couplings $g_{i}(i=3,2, Y)$ are given by those of the SM,

$$
(4 \pi)^{2} \mu \frac{d g_{i}}{d \mu}=b_{i} g_{i}^{3}, \quad\left(\mu<R^{-1}\right)
$$

with $b_{3}=-7, b_{2}=-\frac{19}{6}$ and $b_{Y}=\frac{41}{6}$. Above $R^{-1} \mathrm{QCD}$ should be replaced by the $S U(3)_{1} \times S U(3)_{2}$ gauge interaction. We also need to take into account contributions of KK modes in $\mu \geq R^{-1}$. Since the KK modes heavier than the renormalization scale $\mu$ are decoupled in the 
MS-RGEs, we only need summing up the loops of the KK modes lighter than $\mu$. We estimate the total number of KK modes below $\mu$ by the volume of the momentum space of extra dimensions dividing by the identification factor $n$,

$$
N_{\mathrm{KK}}(\mu)=\frac{\pi(\mu R)^{2}}{n}, \quad\left(\mu \gg R^{-1}\right) .
$$

Note that we impose additional BC's such as Eq. (II.28) other than the BC's for the $T^{2} / Z_{4}$ compactification. Therefore our model corresponds to the case of

$$
n=8 .
$$

The estimate (III.3) works well for $\mu R \gg 1$. (See, e.g. Ref. [15]. ) Within the truncated KK effective theory, we obtain the RGE

$$
(4 \pi)^{2} \mu \frac{d g}{d \mu}=N_{\mathrm{KK}}(\mu) b_{\mathrm{tc}} g^{3}, \quad\left(\mu \geq R^{-1}\right)
$$

with

$$
b_{\mathrm{tc}}=-\frac{22}{3}+\frac{4}{3} \cdot N_{X}, \quad \text { for } \quad S U(3)_{1},
$$

where $N_{X}$ is the number of $\psi_{X}$ with the fundamental representation. Other RGE coefficients are given by

$$
\begin{aligned}
& b^{\prime}=-\frac{14}{3}, \quad \text { for } \quad S U(3)_{2}, \\
& b_{2}^{\prime}=\frac{4}{3}+\frac{1}{6} n_{h}, \quad \text { for } \quad S U(2)_{W}, \\
& b_{Y}^{\prime}=\frac{40}{3}+\frac{1}{6} n_{h}, \quad \text { for } \quad U(1)_{Y} .
\end{aligned}
$$

In the following analysis, we assume that one composite Higgs doublet appears in the low-energy spectrum, i.e., $n_{h}=1$.

When the RG flow of $S U(3)_{1}$ is "walking" more slowly than that of $S U(3)_{2}$, the top condensation is favored rather than the up and charm condensations. Thus we require $b_{\mathrm{tc}}>b^{\prime}$, i.e.,

$$
N_{X} \geq 3 .
$$

We now define the dimensionless bulk gauge coupling $\hat{g}$ as $\hat{g}^{2} \equiv g_{6 D}^{2} \mu^{2}$ and thereby obtain

$$
\hat{g}^{2}(\mu)=(\pi R \mu)^{2} g^{2}(\mu),
$$

where we used Eq. (II.41). Combining Eq. (III.11) with the RGE (III.5), we find RGEs for the dimensionless bulk Topcolor coupling $\hat{g}$,

$$
\mu \frac{d}{d \mu} \hat{g}=\hat{g}+\Omega_{\mathrm{NDA}} b_{\mathrm{tc}} \hat{g}^{3},
$$

with $\Omega_{\mathrm{NDA}}$ being the $D$-dimensional loop factor in the naive dimensional analysis (NDA),

$$
\Omega_{\mathrm{NDA}} \equiv \frac{1}{(4 \pi)^{D / 2} \Gamma(D / 2)} .
$$

$\mu \mathrm{R}$

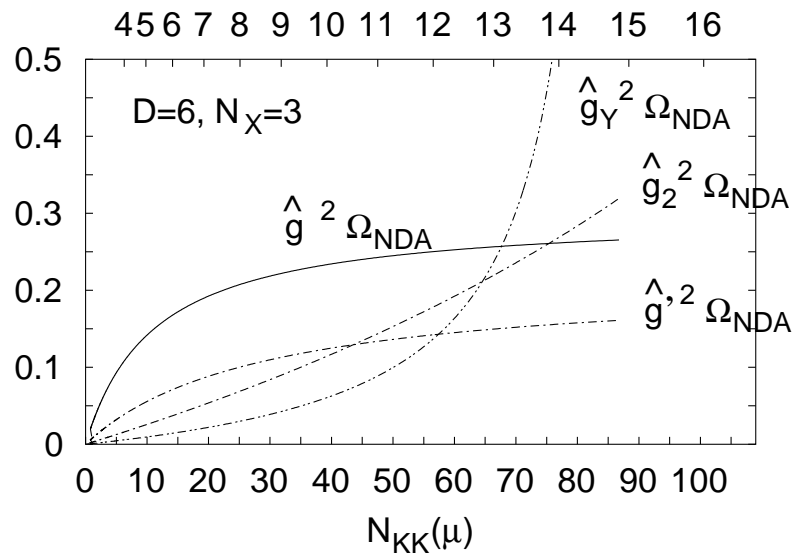

FIG. 1: Typical RG flows for the model with $N_{X}=3$. The ratio of the Topcolor and QCD coupling constants at $R^{-1}=$ $10 \mathrm{TeV}$ is taken to $g^{2}\left(R^{-1}\right) / g_{3}^{2}\left(R^{-1}\right)=4.5$.

The RGEs for $S U(3)_{2}, S U(2)_{W}$, and $U(1)_{Y}$ are the same as Eq. (III.12).

Once we specify $N_{X}$ and the Topcolor coupling at $R^{-1}$, the RG flow of $\hat{g}^{2}$ is completely determined. (See also Eq. (II.44). ) We show typical RG flows in Fig. 11 We used the following values of $\alpha_{i}\left(\equiv g_{i}^{2} /(4 \pi)\right)$ at $\mu=M_{Z}(=$ 91.1876 GeV) as inputs of RGEs: [50]

$$
\begin{aligned}
\alpha_{3}\left(M_{Z}\right) & =0.1172, \\
\alpha_{2}\left(M_{Z}\right) & =0.033822, \\
\alpha_{Y}\left(M_{Z}\right) & =0.010167 .
\end{aligned}
$$

We also note the value of $\alpha_{3}$ at $R^{-1}=10 \mathrm{TeV}$ evolved by the 1-loop RGE,

$$
\alpha_{3}(10 \mathrm{TeV})=0.07264 .
$$

The $U(1)_{Y}$ gauge interaction has the Landau pole $\Lambda_{L Y}$ at which the gauge coupling diverges. (See Fig. 1 ) The bulk gauge coupling $\hat{g}_{Y}(\mu)$ rapidly grows due to the power-like behavior of the running. As a result, the Landau pole $\Lambda_{L Y}$ is not so far from the compactification scale $R^{-1}$. We thus need to introduce a cutoff $\Lambda$ smaller than the Landau pole $\Lambda_{L Y}$.

\section{ANALYSIS OF THE LADDER SD EQUATION}

We explore the energy region where only the top quark condenses while others do not (tMAC region). Since our model explicitly breaks the six dimensional Lorentz symmetry, it is not obvious whether or not the approach of the ladder SD equation for the bulk fermion is appropriate. Nevertheless we may adopt the ladder SD equation in six dimensions, supposing the cutoff $\Lambda R \sim \mathcal{O}(10)$ is large enough. 
The power-like running of the gauge couplings is crucial for the analysis of the tMAC region. Thus we should incorporate the running effects in the ladder SD equation. Several methods have been applied to the phenomenology of the low-energy QCD in four dimensions. Simplest one is the Higashijima-Miransky approximation in which the gauge coupling is replaced by [51]

$$
g^{2} \rightarrow g^{2}\left(\max \left(-p^{2},-q^{2}\right)\right),
$$

where $p$ and $q$ are external and loop momenta of the fermion, respectively. However the HigashijimaMiransky approximation is inconsistent with the axial Ward-Takahashi (WT) identity. A natural choice is to take the argument of $g$ to the gluon loop momentum $(p-q)$,

$$
g^{2} \rightarrow g^{2}\left(-(p-q)^{2}\right) .
$$

In this case, the ladder approximation can be consistent with both of vector and axial WT identities [52]. A demerit of the method is that the angular integration cannot be performed analytically, i.e., the numerical calculation becomes complicated. In Ref. [53], it is shown that the approximation

$$
g^{2} \rightarrow g^{2}\left(-\left(p^{2}+q^{2}\right)\right)
$$

works well in four dimensions. We may adopt Eq. (IV.3. even in extra dimensions.

Let us solve the ladder SD equation including running effects. For consistency with the vector Ward-Takahashi identity, we choose the Landau gauge and then obtain the ladder SD equation for the fermion mass function $B_{f}$ as follows:

$$
\begin{aligned}
B_{f}(x)=(D-1) & \int_{R^{-2}}^{\Lambda^{2}} d y y^{D / 2-1} \frac{B_{f}(y)}{y+B_{f}^{2}(y)} \frac{\kappa_{f}(x+y)}{x+y} \\
& \times\left[K_{B}(x, y)+(x \leftrightarrow y)\right], \quad(\mathrm{IV} .4
\end{aligned}
$$

with $f=t, b, c, u, \ell$, and $x \equiv-p^{2}$, and $y \equiv-q^{2}$, where the kernel $K_{B}$ is given by $[\underline{6}]$

$$
K_{B}(x, y)=\frac{1}{x}\left(1-\frac{y}{3 x}\right) \theta(x-y), \quad \text { for } D=6 .
$$

We identified the infrared (IR) cutoff of the SD equation to the compactification scale $R^{-1}$. The binding strengths $\kappa_{f}$ 's are

$$
\begin{aligned}
& \kappa_{t}\left(\mu^{2}\right)=C_{F} \hat{g}^{2}(\mu) \Omega_{\mathrm{NDA}}+\frac{1}{9} \hat{g}_{Y}^{2}(\mu) \Omega_{\mathrm{NDA}}, \\
& \kappa_{b}\left(\mu^{2}\right)=C_{F} \hat{g}^{2}(\mu) \Omega_{\mathrm{NDA}}-\frac{1}{18} \hat{g}_{Y}^{2}(\mu) \Omega_{\mathrm{NDA}}, \\
& \kappa_{c, u}\left(\mu^{2}\right)=C_{F} \hat{g}^{\prime 2}(\mu) \Omega_{\mathrm{NDA}}+\frac{1}{9} \hat{g}_{Y}^{2}(\mu) \Omega_{\mathrm{NDA}}, \\
& \kappa_{\ell}\left(\mu^{2}\right)= \\
& \frac{1}{2} \hat{g}_{Y}^{2}(\mu) \Omega_{\mathrm{NDA}},
\end{aligned}
$$

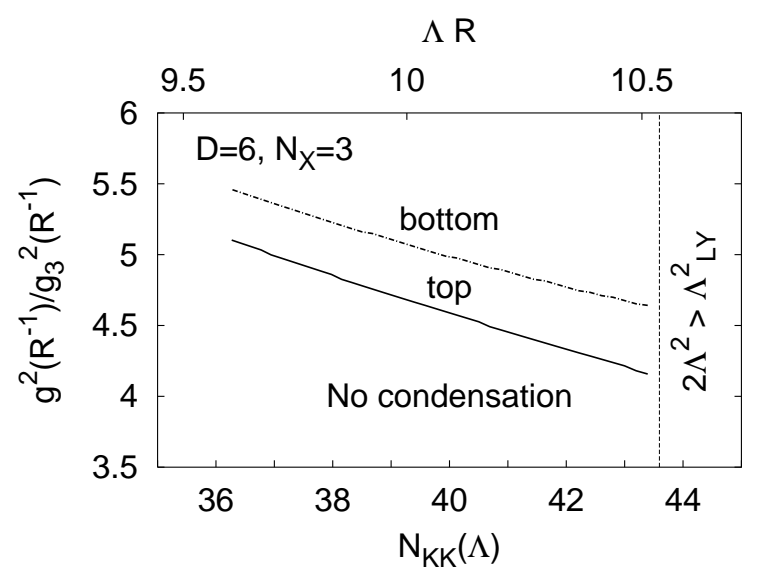

FIG. 2: The tMAC region for the model with $N_{X}=3$. The coupling constants of the Topcolor and QCD at the compactification scale $R^{-1}$ are represented as $g^{2}\left(R^{-1}\right)$ and $g_{3}^{2}\left(R^{-1}\right)$, respectively. In the "top" region only the top condensation occurs (tMAC region). In the "bottom" region the bottom quark condenses as well. No condensation takes place in the region of "No condensation". For $2 \Lambda^{2}>\Lambda_{L Y}^{2}$ the argument of the gauge coupling in the kernel of the ladder SD equation exceeds the Landau pole of $U(1)_{Y}$.

for the top, bottom, charm, up and lepton condensates, respectively. The constant $C_{F}(=4 / 3)$ is the quadratic Casimir of the fundamental representation of $S U(3)$. In the following analysis, we study these four channels. The argument of $\kappa_{f}$ should be smaller than the Landau pole of $U(1)_{Y}$, i.e.,

$$
\max (x+y)=2 \Lambda^{2}<\Lambda_{L Y}^{2} .
$$

We numerically solve the SD equation by using the iteration method, whose details are described in Ref. [6]. In the analysis, we fix the compactification scale $R^{-1}$ to $10 \mathrm{TeV}$. For other values, the results are essentially unchanged. We depict the result for the models with $N_{X}=3$ in Fig. 2] The "top" region in Fig. 2 corresponds to the tMAC. If we choose the ratio of the values of the Topcolor and QCD couplings at $R^{-1}=10 \mathrm{TeV}$ to $g^{2}\left(R^{-1}\right) / g_{3}^{2}\left(R^{-1}\right) \sim 4.2-4.6$, the tMAC region is $\Lambda R \sim 10-10.5$. In the region it turns out that the upand charm-condensations do not occur. For $\Lambda R>10.5$ the lepton condensation is favored.

Similarly, the tMAC regions are also found for models with $N_{X}=4,5$. However the regions become narrower: for $g^{2}\left(R^{-1}\right) / g_{3}^{2}\left(R^{-1}\right) \sim 2.1-2.3, \Lambda R \sim 10.2-10.5$, $\left(N_{X}=4\right)$; for $g^{2}\left(R^{-1}\right) / g_{3}^{2}\left(R^{-1}\right) \sim 1.3-1.4, \Lambda R \sim 10.3-$ $10.5,\left(N_{X}=5\right)$.

\section{SUMMARY AND DISCUSSIONS}

We studied the Topcolor model in the six dimensional bulk. We assigned the nontrivial BC's to the Topcolor gauge fields so that the Topcolor is broken down on the 
boundaries. As a three generation model we considered the model whose charge assignments are shown in Table II Since the top and bottom quarks have the Topcolor charge while the other quarks do not in the model, the up and charm condensations are unlikely to occur. When the bulk $U(1)_{Y}$ interaction is sufficiently strong, the bottom condensation is also suppressed. In this way, we can expect that only the top quark condenses, which is required for a viable model. In order to demonstrate the existence of such a situation, we analyzed the ladder SD equation including the RGE effects of the bulk gauge couplings. We then found that the situation can be realized in the "top" region shown in Fig. 2] which is the result for the model with three extra (heavy) vectorlike fermions having the Topcolor charge, i.e., $N_{X}=3$. For example, when the ratio of the couplings of Topcolor and QCD is taken to $g^{2}\left(R^{-1}\right) / g_{3}^{2}\left(R^{-1}\right) \sim 4.2-4.6$ with $\left.R^{-1} \approx 10 \mathrm{TeV}\right)$ being the compactification scale, the cutoff $\Lambda$ should be $\Lambda R \sim 10-10.5$. The models with $N_{X}=4,5$ may be possible as well.

The electroweak gauge sector of the model is the same as the universal extra dimension model [42]. The compactification scale $R^{-1}$ is severely constrained by the LEP precision data [50]. Since the KK modes of bulk fermions are vector-like, the constraint from the $S$ parameter is suppressed, $S \approx 10^{-2} \sum_{j, k} \frac{m_{t}^{2}}{M_{j, k}^{2}}$. Hence the $T$-parameter constraint is essential. We may estimate the $T$-parameter as in Ref. [42],

$$
T \approx 0.76 \sum_{j, k} \frac{m_{t}^{2}}{M_{j, k}^{2}},
$$

where we neglected $\mathcal{O}\left(m_{t}^{4} / M_{j, k}^{4}\right)$ contributions. When we take $\max \left(M_{j, k}\right)=\Lambda$ or $\sqrt{2} \Lambda$ with $\Lambda \sim(10-10.5) R^{-1}$, the estimate of the $T$-parameter is

$$
T \approx(4-5) \times 10^{-2} \frac{\left(1 \mathrm{TeV}^{2}\right)}{R^{-2}\left(\mathrm{TeV}^{2}\right)} .
$$

The current constraint $T<0.02$ at $95 \%$ C.L. with the Higgs boson mass $m_{H}=117 \mathrm{GeV}[50]$ yields $R^{-1}>$ $1.4-1.6 \mathrm{TeV}$. For larger $m_{H}$ the lower bound of $R^{-1}$ gets smaller. For the reference value $R^{-1}=10 \mathrm{TeV}$, we can expect that the contributions of KK modes to the $T$-parameter is negligibly small, even if we take into account errors arising from nonperturbative effects. In this case, however, we may need to allow the fine tuning of $\mathcal{O}\left(m_{t}^{2} R^{2}\right) \sim 3 \times 10^{-4}$.

Our approach is very sensitive to the cutoff, i.e., the UV physics. The UV completion by theory space [54, 55] may be required.

\section{Acknowledgments}

We are thankful to J. Minahan and V. Miransky for useful comments. The work is supported by KRF PBRG
2002-070-C00022 and also in part by funds provided by the U.S. Department of Energy (D.O.E.) under cooperative research agreement \#DF-FC02-94ER40818.

\section{APPENDIX A: SQUARE COMPACTIFICATION}

We summarize the chiral compactification on the square with $0 \leq y^{5}, y^{6} \leq L$. Following Dobrescu and Pontón [43], we identify two adjacent sides as

$$
(y, 0) \equiv(0, y), \quad(y, L) \equiv(L, y), \quad{ }^{\forall} y \in[0, L],
$$

which is closely related to the orbifold compactification on $T^{2} / Z_{4}$. We include a gauge field as well as a chiral fermion in the bulk. Here we argue the bulk fermion with positive chirality. It is straightforward to extend the arguments to fermions with negative chirality.

Let us study the Lagrangian,

$$
\mathcal{L}=\mathcal{L}_{\psi_{+}}+\mathcal{L}_{\text {gauge }}
$$

with

$$
\mathcal{L}_{\psi_{+}}=\bar{\psi}_{+} i D_{M} \Gamma^{M} \psi_{+}
$$

and

$$
\mathcal{L}_{\text {gauge }}=-\frac{1}{4} F_{M N}^{a} F^{a M N}
$$

where $M=0,1,2,3,5,6$,

$$
D_{M} \equiv \frac{1}{2} \stackrel{\leftrightarrow}{\partial}_{M}-i g_{6 D} A_{M},
$$

and

$$
F_{M N}^{a} \equiv \partial_{M} A_{N}^{a}-\partial_{N} A_{M}^{a}+g_{6 D} f^{a b c} A_{M}^{b} A_{N}^{c} .
$$

The chiral fermions $\psi_{ \pm}$in the bulk are defined as

$$
\psi_{ \pm} \equiv P_{ \pm} \psi
$$

with the chiral projection operators $P_{ \pm}$,

$$
P_{ \pm} \equiv \frac{1}{2}\left(1 \pm \Gamma_{\chi, 7}\right)
$$

where the chirality matrix $\Gamma_{\chi, 7}$ in six dimensions is

$$
\Gamma_{\chi, 7} \equiv \Gamma^{0} \Gamma^{1} \Gamma^{2} \Gamma^{3} \Gamma^{5} \Gamma^{6}, \quad \Gamma_{\chi, 7} \Gamma_{\chi, 7}=1 .
$$

Hence the fermions $\psi_{ \pm}$have four components. For our purpose, it is convenient to use four dimensional right/left-handed notations. The four dimensional chirality matrix $\Gamma_{\chi, 5}$ is

$$
\Gamma_{\chi, 5} \equiv i \Gamma^{0} \Gamma^{1} \Gamma^{2} \Gamma^{3}, \quad \Gamma_{\chi, 5} \Gamma_{\chi, 5}=1 .
$$

The matrices $\Gamma_{\chi, 5}$ and $\Gamma_{\chi, 7}$ satisfy

$$
\left[\Gamma_{\chi, 5}, \Gamma_{\chi, 7}\right]=0,
$$


so that $\Gamma_{\chi, 5}$ and $\Gamma_{\chi, 7}$ are simultaneously diagonalizable. Thus we further decompose $\psi_{ \pm}$into the four dimensional right/left-handed fermions:

$$
\psi_{ \pm}=\psi_{ \pm R}+\psi_{ \pm L}
$$

where

$$
\psi_{ \pm R} \equiv P_{R} \psi_{ \pm}, \quad \psi_{ \pm L} \equiv P_{L} \psi_{ \pm}
$$

with the four dimensional chiral projection operators $P_{R, L}$

$$
P_{R, L} \equiv \frac{1}{2}\left(1 \pm \Gamma_{\chi, 5}\right)
$$

Noting

$$
\left\{\Gamma^{\mu}, \Gamma_{\chi, 5}\right\}=0, \quad \text { for } \quad \mu=0,1,2,3
$$

and

$$
\left[\Gamma^{m}, \Gamma_{\chi, 5}\right]=0, \quad \text { for } \quad m=5,6,
$$

the Lagrangian $\mathcal{L}_{\psi_{+}}$is rewritten in terms of $\psi_{+R}$ and $\psi_{+L}$ as follows:

$$
\mathcal{L}_{\psi_{+}}=\mathcal{L}_{R R+L L}+\mathcal{L}_{R L+L R}
$$

with

$$
\mathcal{L}_{R R+L L} \equiv \bar{\psi}_{+R} i D_{\mu} \Gamma^{\mu} \psi_{+R}+\bar{\psi}_{+L} i D_{\mu} \Gamma^{\mu} \psi_{+L},
$$

and

$$
\mathcal{L}_{R L+L R} \equiv \bar{\psi}_{+R} i D_{m} \Gamma^{m} \psi_{+L}+\bar{\psi}_{+L} i D_{m} \Gamma^{m} \psi_{+R} .
$$

Under the identification (A.1), the Lagrangian should be the same:

$$
\left.\mathcal{L}\right|^{(y, 0)}=\left.\mathcal{L}\right|^{(0, y)},\left.\quad \mathcal{L}\right|^{(y, L)}=\left.\mathcal{L}\right|^{(L, y)} .
$$

We then impose the BC's on fermions as

$$
\begin{aligned}
& \psi_{+R}(y, 0)=e^{\frac{i \pi}{2} n} \psi_{+R}(0, y), \\
& \psi_{+L}(y, 0)=i e^{\frac{i \pi}{2} n} \psi_{+L}(0, y),
\end{aligned}
$$

and

$$
\begin{aligned}
& \psi_{+R}(y, L)=(-1)^{\ell} e^{\frac{i \pi}{2} n} \psi_{+R}(L, y), \\
& \psi_{+L}(y, L)=i(-1)^{\ell} e^{\frac{i \pi}{2} n} \psi_{+L}(L, y),
\end{aligned}
$$

where the integers $n$ and $\ell$ can take the values of $n=$ $0,1,2,3$ and $\ell=0,1$, respectively. Differentiating the BC's A.21 - A.22 with respect to $y$, we find

$$
\begin{aligned}
& \partial_{5} \psi_{+R}(y, 0)=e^{\frac{i \pi}{2} n} \partial_{6} \psi_{+R}(0, y), \\
& \partial_{5} \psi_{+L}(y, 0)=i e^{\frac{i \pi}{2} n} \partial_{6} \psi_{+L}(0, y),
\end{aligned}
$$

and

$$
\begin{aligned}
& \partial_{5} \psi_{+R}(y, L)=(-1)^{\ell} e^{\frac{i \pi}{2} n} \partial_{6} \psi_{+R}(L, y), \\
& \partial_{5} \psi_{+L}(y, L)=i(-1)^{\ell} e^{\frac{i \pi}{2} n} \partial_{6} \psi_{+L}(L, y) .
\end{aligned}
$$

We further impose the BC's on the derivative terms as

$$
\begin{aligned}
& \partial_{6} \psi_{+R}(y, 0)=-e^{\frac{i \pi}{2} n} \partial_{5} \psi_{+R}(0, y), \\
& \partial_{6} \psi_{+L}(y, 0)=-i e^{\frac{i \pi}{2} n} \partial_{5} \psi_{+L}(0, y),
\end{aligned}
$$

and

$$
\begin{aligned}
& \partial_{6} \psi_{+R}(y, L)=(-1)^{\ell+1} e^{\frac{i \pi}{2} n} \partial_{5} \psi_{+R}(L, y), \\
& \partial_{6} \psi_{+L}(y, L)=i(-1)^{\ell+1} e^{\frac{i \pi}{2} n} \partial_{5} \psi_{+L}(L, y) .
\end{aligned}
$$

The BC's of the derivative terms imply the identification of gauge bosons as

$$
\begin{array}{ll}
A_{\mu}(y, 0)=A_{\mu}(0, y), & A_{\mu}(y, L)=A_{\mu}(L, y) \\
A_{5}(y, 0)=A_{6}(0, y), & A_{5}(y, L)=A_{6}(L, y) \\
A_{6}(y, 0)=-A_{5}(0, y), & A_{6}(y, L)=-A_{5}(L, y)
\end{array}
$$

We differentiate Eq. (A.27) with respect to $y$ and find

$$
\begin{aligned}
& \left.\partial_{5} A_{\mu}\right|^{(y, 0),(y, L)}=\left.\partial_{6} A_{\mu}\right|^{(0, y),(L, y)}, \\
& \left.\partial_{5} A_{6}\right|^{(y, 0),(y, L)}=-\left.\partial_{6} A_{5}\right|^{(0, y),(L, y)} .
\end{aligned}
$$

The identification A.20 for the gauge sector $\mathcal{L}_{\text {gauge }}$ then requires the BC's

$$
\begin{gathered}
\left.\partial_{6} A_{\mu}\right|^{(y, 0),(y, L)}=-\left.\partial_{5} A_{\mu}\right|^{(0, y),(L, y)}, \\
\left.\partial_{6} A_{5}\right|^{(y, 0),(y, L)}=-\left.\partial_{5} A_{6}\right|^{(0, y),(L, y)} .
\end{gathered}
$$

Now it is easy to check that the identification A.20 is satisfied. From the BC's (A.21)-A.22, $\mathcal{L}_{R R+L L}$ defined by Eq. A.18) is obviously identical to the reflection under Eq. A.1). To see the identity for $\mathcal{L}_{R L+L R}$, we apply the relations

$$
\Gamma^{5} P_{R} P_{ \pm}= \pm i \Gamma^{6} P_{R} P_{ \pm}, \quad \Gamma^{5} P_{L} P_{ \pm}=\mp i \Gamma^{6} P_{L} P_{ \pm},
$$

and then find

$$
\begin{aligned}
\mathcal{L}_{R L+L R}= & \bar{\psi}_{+R}\left(D_{5} \Gamma^{6}-D_{6} \Gamma^{5}\right) \psi_{+L} \\
& +\bar{\psi}_{+L}\left(-D_{5} \Gamma^{6}+D_{6} \Gamma^{5}\right) \psi_{+R} .
\end{aligned}
$$

By using the BC's of Eqs. A.21 A.26, we can easily confirm the identification $\left.\mathcal{L}_{\psi_{+}}\right|^{(y, 0),(y, L)}=$ $\left.\mathcal{L}_{\psi_{+}}\right|^{(0, y),(L, y)}$. How about the identification of the gauge sector? The derivative of Eq. A.27) with respect to $x^{\mu}$ and Eqs. A.28)-(A.29) yield

$$
\begin{aligned}
& \left.F_{\mu \nu}^{a}\right|^{(y, 0),(y, L)}=\left.F_{\mu \nu}^{a}\right|^{(0, y),(L, y)}, \\
& \left.F_{\mu 5}^{a}\right|^{(y, 0),(y, L)}=\left.F_{\mu 6}^{a}\right|^{(0, y),(L, y)}, \\
& \left.F_{\mu 6}^{a}\right|^{(y, 0),(y, L)}=-\left.F_{\mu 5}^{a}\right|^{(0, y),(L, y)}, \\
& \left.F_{56}^{a}\right|^{(y, 0),(y, L)}=\left.F_{56}^{a}\right|^{(0, y),(L, y)},
\end{aligned}
$$

so that the identification $\left.\mathcal{L}_{\text {gauge }}\right|^{(y, 0),(y, L)}=$ $\left.\mathcal{L}_{\text {gauge }}\right|^{(0, y),(L, y)}$ is clearly satisfied. 
We can show that the phase factor should be $e^{\frac{i \pi}{2} n}(n=$ $0,1,2,3)$ after some algebraic calculation. [43] We will not repeat it here. In this paper, we take the convention

$$
n=0, \quad \ell=0 \text {. }
$$

The BC's yield the Kaluza-Klein (KK) decomposition of the gauge field $A_{\mu}$ :

$$
\begin{aligned}
A_{\mu}\left(x^{\mu}, y^{5}, y^{6}\right) & =\frac{1}{L} \sum_{j \geq k \geq 0} A_{\mu, c c}^{[j, k]}\left(x^{\mu}\right) f_{c c}^{[j, k]} \\
& +\frac{1}{L} \sum_{j>k>0} A_{\mu, s s}^{[j, k]}\left(x^{\mu}\right) f_{s s}^{[j, k]}
\end{aligned}
$$

with

$$
\begin{array}{r}
f_{c c}^{[0,0]} \equiv 1, \quad f_{c c}^{[j \neq 0,0]} \equiv \cos \left(\frac{\pi}{L} j y^{5}\right)+\cos \left(\frac{\pi}{L} j y^{6}\right) \\
f_{c c}^{[j, j]} \equiv 2 \cos \left(\frac{\pi}{L} j y^{5}\right) \cos \left(\frac{\pi}{L} j y^{6}\right),(j \neq 0) \\
f_{c c}^{[j, k]} \equiv \sqrt{2}\left[\cos \left(\frac{\pi}{L} j y^{5}\right) \cos \left(\frac{\pi}{L} k y^{6}\right)\right. \\
\left.+\cos \left(\frac{\pi}{L} k y^{5}\right) \cos \left(\frac{\pi}{L} j y^{6}\right)\right],(j>k>0)
\end{array}
$$

and

$$
\begin{array}{r}
f_{s s}^{[j, k]} \equiv-\sqrt{2}\left[\sin \left(\frac{\pi}{L} j y^{5}\right) \sin \left(\frac{\pi}{L} k y^{6}\right)\right. \\
\left.-\sin \left(\frac{\pi}{L} k y^{5}\right) \sin \left(\frac{\pi}{L} j y^{6}\right)\right] .
\end{array}
$$

The KK expansions of $\psi_{+R}$ and $\psi_{+L}$ are given by

$$
\begin{aligned}
\psi_{+R}\left(x^{\mu}, y^{5}, y^{6}\right) & =\frac{1}{L} \sum_{j \geq k \geq 0} \psi_{+R, c c}^{[j, k]}\left(x^{\mu}\right) f_{c c}^{[j, k]} \\
& +\frac{1}{L} \sum_{j>k>0} \psi_{+R, s s}^{[j, k]}\left(x^{\mu}\right) f_{s s}^{[j, k]},
\end{aligned}
$$

and

$$
\begin{aligned}
\psi_{+L}\left(x^{\mu}, y^{5}, y^{6}\right) & =\frac{1}{L} \sum_{j \geq k \geq 0} \psi_{+L, 1}^{[j, k]}\left(x^{\mu}\right) g_{1}^{[j, k]} \\
& +\frac{1}{L} \sum_{j>k>0} \psi_{+L, 2}^{[j, k]}\left(x^{\mu}\right) g_{2}^{[j, k]}
\end{aligned}
$$

with

$$
g_{1}^{[j, k]} \equiv \frac{j}{\sqrt{j^{2}+k^{2}}} f_{s c}^{[j, k]}-\frac{i k}{\sqrt{j^{2}+k^{2}}} f_{c s}^{[j, k]},
$$

and

$$
g_{2}^{[j, k]} \equiv-\frac{i k}{\sqrt{j^{2}+k^{2}}} f_{s c}^{[j, k]}+\frac{j}{\sqrt{j^{2}+k^{2}}} f_{c s}^{[j, k]},
$$

where

$$
\begin{gathered}
f_{s c}^{[j, k]} \equiv \sqrt{\frac{2}{1+\delta_{k, 0}}\left[\sin \left(\frac{\pi}{L} j y^{5}\right) \cos \left(\frac{\pi}{L} k y^{6}\right)\right.} \\
\left.-i \cos \left(\frac{\pi}{L} k y^{5}\right) \sin \left(\frac{\pi}{L} j y^{6}\right)\right]
\end{gathered}
$$

and

$$
\begin{gathered}
f_{c s}^{[j, k]} \equiv \sqrt{\frac{2}{1+\delta_{j, 0}}}\left[\cos \left(\frac{\pi}{L} j y^{5}\right) \sin \left(\frac{\pi}{L} k y^{6}\right)\right. \\
\left.+i \sin \left(\frac{\pi}{L} k y^{5}\right) \cos \left(\frac{\pi}{L} j y^{6}\right)\right]
\end{gathered}
$$

The KK decompositions of $\psi_{-L}$ and $\psi_{-R}$ are the same as those of $\psi_{+R}$ and $\psi_{+L}$ :

$$
\begin{aligned}
\psi_{-L}\left(x^{\mu}, y^{5}, y^{6}\right) & =\frac{1}{L} \sum_{j \geq k \geq 0} \psi_{-L, c c}^{[j, k]}\left(x^{\mu}\right) f_{c c}^{[j, k]} \\
& +\frac{1}{L} \sum_{j>k>0} \psi_{-L, s s}^{[j, k]}\left(x^{\mu}\right) f_{s s}^{[j, k]},
\end{aligned}
$$

and

$$
\begin{aligned}
\psi_{-R}\left(x^{\mu}, y^{5}, y^{6}\right) & =\frac{1}{L} \sum_{j \geq k \geq 0} \psi_{-R, 1}^{[j, k]}\left(x^{\mu}\right) g_{1}^{[j, k]} \\
& +\frac{1}{L} \sum_{j>k>0} \psi_{-R, 2}^{[j, k]}\left(x^{\mu}\right) g_{2}^{[j, k]}
\end{aligned}
$$

In this way, the chiral compactification on the square leaves the zero modes $A_{\mu, c c}^{[0,0]}, \psi_{+R, c c}^{[0,0]}$, and $\psi_{-L, c c}^{[0,0]}$.

\section{APPENDIX B: GAUGE SYMMETRY ON BOUNDARIES}

We study the gauge symmetry on the boundaries. The four/five dimensional notations are more convenient than the six dimensional one. We thus rewrite $\mathcal{L}_{\psi_{+}}$by using the following representation of the gamma matrices,

$$
\Gamma^{\mu}=\left(\begin{array}{cc}
\gamma^{\mu} & 0 \\
0 & \gamma^{\mu}
\end{array}\right)
$$

$$
\begin{aligned}
\Gamma^{5} & =\left(\begin{array}{cc}
0 & i \gamma_{5} \\
i \gamma_{5} & 0
\end{array}\right), \\
\Gamma^{6} & =\left(\begin{array}{cc}
0 & \gamma_{5} \\
-\gamma_{5} & 0
\end{array}\right),
\end{aligned}
$$

where $\gamma^{\mu}$ and $\gamma_{5}$ are $4 \times 4$ matrices. We take the spacetime metric to $\operatorname{diag}(+,-,-, \cdots,-)$, so that the five dimensional gamma matrices are $\gamma^{\mu}, i \gamma_{5}$. Noting that

$$
\Gamma_{\chi, 7} \equiv \Gamma^{0} \Gamma^{1} \Gamma^{2} \Gamma^{3} \Gamma^{5} \Gamma^{6}=\left(\begin{array}{cc}
-\gamma_{5} & 0 \\
0 & \gamma_{5}
\end{array}\right)
$$


the chiral fermions $\psi_{+L,+R}$ should be

$$
\psi_{+L} \rightarrow\left(\begin{array}{c}
\psi_{+L} \\
0
\end{array}\right), \quad \psi_{+R} \rightarrow\left(\begin{array}{c}
0 \\
\psi_{+R}
\end{array}\right) .
$$

In the four/five dimensional notations, the Lagrangian $\mathcal{L}_{\psi_{+}}\left(=\mathcal{L}_{R R+L L}+\mathcal{L}_{R L+L R}\right)$ is represented as

$$
\mathcal{L}_{R R+L L}=\bar{\psi}_{+R} i D_{\mu} \gamma^{\mu} \psi_{+R}+\bar{\psi}_{+L} i D_{\mu} \gamma^{\mu} \psi_{+L},
$$

and

$$
\begin{aligned}
\mathcal{L}_{R L+L R}= & \bar{\psi}_{+R}\left[i D_{5}-D_{6}\right]\left(i \gamma_{5}\right) \psi_{+L} \\
& +\bar{\psi}_{+L}\left[i D_{5}+D_{6}\right]\left(i \gamma_{5}\right) \psi_{+R} .
\end{aligned}
$$

By performing integration by parts, we obtain the $R L+$ $L R$ part of the action,

$$
\begin{aligned}
& S_{R L+L R}=\int d x^{4} \int d y^{5} d y^{6} \mathcal{L}_{R L+L R}^{\prime} \\
& \left.+\frac{1}{2} \int d x^{4} \int d y^{6}\left[\left(\bar{\psi}_{+R} \psi_{+L}\right)+\text { (h.c. }\right)\right]_{\left(0, y^{6}\right)}^{\left(L, y^{6}\right)} \\
& \left.+\frac{1}{2} \int d x^{4} \int d y^{5}\left[i\left(\bar{\psi}_{+R} \psi_{+L}\right)+\text { (h.c. }\right)\right]_{\left(y^{5}, 0\right)}^{\left(y^{5}, L\right)}
\end{aligned}
$$

with

$$
\begin{aligned}
& \mathcal{L}_{R L+L R}^{\prime} \equiv\left(-i \partial_{5}+\partial_{6}\right) \bar{\psi}_{+R}\left(i \gamma_{5}\right) \psi_{+L} \\
& \quad+\bar{\psi}_{+R}\left[g_{6 D} A_{5}+i g_{6 D} A_{6}\right]\left(i \gamma_{5}\right) \psi_{+L} \\
& \quad+\bar{\psi}_{+L}\left(i \partial_{5}+\partial_{6}+g_{6 D} A_{5}-i g_{6 D} A_{6}\right)\left(i \gamma_{5}\right) \psi_{+R} .
\end{aligned}
$$

The surface terms in Eq. (B.8) are vanishing thanks to the BC's A.21 - A.22. Therefore we may use $\mathcal{L}_{R L+L R}^{\prime}$ instead of $\mathcal{L}_{R L+L R}$.

Now we impose the BC's in order to restore the five dimensional gauge symmetry on the boundaries. Since the gauge scalars should be massive, it is natural to assign the Dirichlet BC's to $A_{5}$ and $A_{6}$, i.e.,

$$
\left.A_{5}\right|^{(0, y),(L, y)}=0,\left.\quad A_{6}\right|^{(y, 0),(y, L)}=0 .
$$

Then the derivative term of $\psi_{+R}$ should be zero simultaneously from Eq. (B.9),

$$
\left.\partial_{5} \psi_{+R}\right|^{(0, y),(L, y)}=0,\left.\quad \partial_{6} \psi_{+R}\right|^{(y, 0),(y, L)}=0 .
$$

In order to ensure nonvanishing $\mathcal{L}_{R L+L R}^{\prime}$, we impose

$$
\left.\left(\partial_{5}+i \partial_{6}\right) \psi_{+L}\right|^{(0, y),(L, y),(y, 0),(y, L)} \neq 0 .
$$

The BC's for the gauge bosons are easily found as

$$
\left.\partial_{5} A_{\mu}\right|^{(0, y),(L, y)}=0,\left.\quad \partial_{6} A_{\mu}\right|^{(y, 0),(y, L)}=0,
$$

and

$$
\left.\partial_{5} A_{6}\right|^{(0, y),(L, y)}=0,\left.\quad \partial_{6} A_{5}\right|^{(y, 0),(y, L)}=0 .
$$

We did not fix the gauge yet, so that the mixing terms of $A_{\mu} A_{5}$ and $A_{\mu} A_{6}$ remain. For completeness, one may introduce $R_{\xi}$ gauge fixing terms, etc..
[1] I. Antoniadis, Phys. Lett. B246, 377 (1990).

[2] K. R. Dienes, E. Dudas, and T. Gherghetta, Phys. Lett. B436, 55 (1998), arXiv:hep-ph/9803466; Nucl. Phys. B537, 47 (1999), arXiv:hep-ph/9806292.

[3] B. A. Dobrescu, Phys. Lett. B 461, 99 (1999); hep-ph/9903407

[4] H. Cheng, B. A. Dobrescu, and C. T. Hill, Nucl. Phys. B 589, 249 (2000).

[5] N. Arkani-Hamed, H.-C. Cheng, B. A. Dobrescu, and L. J. Hall, Phys. Rev. D62, 096006 (2000).

[6] M. Hashimoto, M. Tanabashi, and K. Yamawaki, Phys. Rev. D64, 056003 (2001), arXiv:hep-ph/0010260.

[7] N. Rius, and V. Sanz, Phys. Rev. D64, 075006 (2001), arXiv:hep-ph/0103086.

[8] H. Abe, K. Fukazawa, and T. Inagaki, Prog. Theor. Phys. 107, 1047 (2002), arXiv:hep-ph/0107125; H. Abe, and T. Inagaki, Phys. Rev. D66, 085001 (2002), arXiv:hep-ph/0206282.

[9] V. A. Miransky, M. Tanabashi, and K. Yamawaki, Phys. Lett. B221, 177 (1989); Mod. Phys. Lett. A4, 1043 (1989).
[10] Y. Nambu, Enrico Fermi Institute Report No. 89-08, 1989 (unpublished); in Proceedings of the 1989 Workshop on Dynamical Symmetry Breaking, edited by T. Muta and K. Yamawaki (Nagoya University, Nagoya, Japan, 1990).

[11] W. J. Marciano, Phys. Rev. Lett. 62, 2793 (1989); Phys. Rev. D41, 219 (1990).

[12] W. A. Bardeen, C. T. Hill, and M. Lindner, Phys. Rev. D41, 1647 (1990).

[13] A. B. Kobakhidze, Phys. Atom. Nucl. 64, 941 (2001), [Yad. Fiz. 64, 1010 (2001)], arXiv:hep-ph/9904203.

[14] V. Gusynin, M. Hashimoto, M. Tanabashi, and K. Yamawaki, Phys. Rev. D65, 116008 (2002), arXiv:hep-ph/0201106.

[15] M. Hashimoto, M. Tanabashi, and K. Yamawaki, Phys. Rev. D69, 076004 (2004), arXiv:hep-ph/0311165.

[16] T. Appelquist, B. A. Dobrescu, E. Ponton and H. U. Yee, Phys. Rev. Lett. 87, 181802 (2001), arXiv:hep-ph/0107056.

[17] B. A. Dobrescu and E. Poppitz, Phys. Rev. Lett. 87, 031801 (2001), arXiv:hep-ph/0102010.

[18] V. P. Gusynin, M. Hashimoto, M. Tanabashi and 
K. Yamawaki, Phys. Rev. D70, 096005 (2004), arXiv:hep-ph/0406194.

[19] C. T. Hill, Phys. Lett. 266 B, 419 (1991).

[20] C. T. Hill, Phys. Lett. B 345, 483 (1995); K. Lane, and E. Eichten, Phys. Lett. B 352, 382 (1995); K. Lane, Phys. Lett. B 433, 96 (1998).

[21] B. A. Dobrescu and C. T. Hill, Phys. Rev. Lett. 81, 2634 (1998), arXiv:hep-ph/9712319; R. S. Chivukula, B. A. Dobrescu, H. Georgi and C. T. Hill, Phys. Rev. D59, 075003 (1999), arXiv:hep-ph/9809470.

[22] G. Cvetic, Rev. Mod. Phys. 71, 513 (1999), arXiv:hep-ph/9702381.

[23] C. T. Hill, and E. H. Simmons, Phys. Rept. 381, 235 (2003), [Erratum ibid. 390, 553 (2004)], arXiv:hep-ph/0203079.

[24] H. C. Cheng, C. T. Hill, and J. Wang, Phys. Rev. D64, 095003 (2001), arXiv:hep-ph/0105323.

[25] H. J. He, C. T. Hill, and T. M. P. Tait, Phys. Rev. D65, 055006 (2002), arXiv:hep-ph/0108041.

[26] Y. Kawamura, Prog. Theor. Phys. 103, 613 (2000), arXiv:hep-ph/9902423; Prog. Theor. Phys. 105, 691 (2001), arXiv:hep-ph/0012352; Prog. Theor. Phys. 105, 999 (2001), arXiv:hep-ph/0012125; and see e.g., A. Hebecker, and J. March-Russell, Nucl. Phys. B613, 3 (2001), arXiv:hep-ph/0106166 ; Nucl. Phys. B625, 128 (2002), arXiv:hep-ph/0107039 ; L. J. Hall, Y. Nomura, T. Okui, and D. R. Smith, Phys. Rev. D65, 035008 (2002), arXiv:hep-ph/0108071; L. J. Hall, and Y. Nomura, Phys. Rev. D65, 125012 (2002), arXiv:hep-ph/0111068; L. J. Hall, and Y. Nomura, Phys. Rev. D66, 075004 (2002), arXiv:hep-ph/0205067.

[27] C. Csaki, C. Grojean, H. Murayama, L. Pilo, and J. Terning, Phys. Rev. D69, 055006 (2004), arXiv:hep-ph/0305237.

[28] C. Csaki, C. Grojean, L. Pilo, and J. Terning, Phys. Rev. Lett. 92, 101802 (2004), arXiv:hep-ph/0308038.

[29] Y. Nomura, JHEP 0311, 050 (2003), arXiv:hep-ph/0309189.

[30] R. Barbieri, A. Pomarol, and R. Rattazzi, Phys. Lett. B591, 141 (2004), arXiv:hep-ph/0310285.

[31] H. Davoudiasl, J. L. Hewett, B. Lillie, and T. G. Rizzo, Phys. Rev. D70, 015006 (2004), arXiv:hep-ph/0312193.

[32] R. Foadi, S. Gopalakrishna, and C. Schmidt, JHEP 0403, 042 (2004), arXiv:hep-ph/0312324.

[33] J. Hirn, and J. Stern, Eur. Phys. J. C34, 447 (2004), arXiv:hep-ph/0401032.
[34] R. S. Chivukula, M. Kurachi, and M. Tanabashi, JHEP 0406, 004 (2004), arXiv:hep-ph/0403112; R. S. Chivukula, E. H. Simmons, H. J. He, M. Kurachi and M. Tanabashi, Phys. Rev. D70, 075008 (2004), arXiv:hep-ph/0406077.

[35] H. Davoudiasl, J. L. Hewett, B. Lillie, and T. G. Rizzo, JHEP 0405, 015 (2004), arXiv:hep-ph/0403300.

[36] S. Gabriel, S. Nandi, and G. Seidl, Phys. Lett. B603, 74 (2004), arXiv:hep-ph/0406020.

[37] N. Evans, and P. Membry, hep-ph/0406285

[38] J. L. Hewett, B. Lillie, and T. G. Rizzo, JHEP 0410, 014 (2004), arXiv:hep-ph/0407059.

[39] M. Papucci, hep-ph/0408058

[40] H. Georgi, Phys. Rev. D71, 015016 (2005), arXiv:hep-ph/0408067.

[41] M. Perelstein, JHEP 0410, 010 (2004), arXiv:hep-ph/0408072.

[42] T. Appelquist, H. C. Cheng and B. A. Dobrescu, Phys. Rev. D64, 035002 (2001), arXiv:hep-ph/0012100.

[43] B. A. Dobrescu, and E. Ponton, JHEP 0403, 071 (2004), arXiv:hep-th/0401032.

[44] B. Holdom, Phys. Lett. B150, 301 (1985).

[45] K. Yamawaki, M. Bando, and K. Matumoto, Phys. Rev. Lett. 56, 1335 (1986);

[46] M. Bando, T. Morozumi, H. So, and K. Yamawaki, Phys. Rev. Lett. 59, 389 (1987).

[47] T. Akiba, and T. Yanagida, Phys. Lett. B169, 432 (1986).

[48] T. Appelquist, D. Karabali, and L.C.R. Wijewardhana, Phys. Rev. Lett. 57, 957 (1986); T. Appelquist, and L. C. R. Wijewardhana, Phys. Rev. D36, 568 (1987).

[49] M. B. Green and J. H. Schwarz, Phys. Lett. 149B, 117 (1984).

[50] S. Eidelman et al. [Particle Data Group Collaboration], Phys. Lett. B592, 1 (2004).

[51] V. A. Miransky, Sov. J. Nucl. Phys. 38, 280 (1983); K. Higashijima, Phys. Rev. D29, 1228 (1984).

[52] T. Kugo, and M. G. Mitchard, Phys. Lett. B282, 162 (1992).

[53] K.-I. Aoki, M. Bando, T. Kugo, and M. G. Mitchard, Prog. Theor. Phys. 85, 355 (1991).

[54] N. Arkani-Hamed, A. G. Cohen, and H. Georgi, Phys. Rev. Lett. 86, 4757 (2001), arXiv:hep-th/0104005.

[55] C. T. Hill, S. Pokorski and J. Wang, Phys. Rev. D64, 105005 (2001), arXiv:hep-th/0104035. 